\title{
Application value of the NoSAS score for screening sleep-disordered breathing
}

\author{
Min Peng ${ }^{1 \#}$, Riken Chen ${ }^{1 \#}$, Junfen Cheng ${ }^{1}$, Jieying Li $^{2}$, Wang Liu ${ }^{1}$, Cheng Hong ${ }^{2}$ \\ ${ }^{1}$ Department of Respiration, the Second Affiliated Hospital of Guangdong Medical University, Zhanjiang 524023, China; ${ }^{2}$ State Key Laboratory of \\ Respiratory Disease, the First Affiliated Hospital of Guangzhou Medical University, Guangzhou Institute of Respiratory Disease, National Clinical \\ Research Center for Respiratory Disease, Guangzhou 510120, China \\ Contributions: (I) Conception and design: M Peng, R Chen; (II) Administrative support: C Hong; (III) Provision of study materials or patients: \\ J Cheng; (IV) Collection and assembly of data: J Li; (V) Data analysis and interpretation: W Liu; (VI) Manuscript writing: All authors; (VII) Final \\ approval of manuscript: All authors. \\ "These authors contributed equally to this work. \\ Correspondence to: Cheng Hong. State Key Laboratory of Respiratory Disease, the First Affiliated Hospital of Guangzhou Medical University, \\ Guangzhou Institute of Respiratory Disease, National Clinical Research Center for Respiratory Disease, 151 Yanjiang Road, Guangzhou 510120, \\ China. Email: gyfyyhc@126.com.
}

Background: Diagnosis of sleep-disordered breathing (SDB) requires overnight polysomnography (PSG). Because of the cost and low availability of these procedures, the NoSAS score was developed to identify subjects at high risk of SDB. To evaluate the clinical utility of the NoSAS score for screening patients with SDB in China and to compare the predictive value of the NoSAS score with the Epworth Sleepiness Scale (ESS), we used the STOP-Bang questionnaire and the Berlin questionnaire.

Methods: In our study, we retrospectively reviewed the existing clinical data of patients who underwent an overnight PSG for suspected SDB from June 2014 to September 2017 at the sleep medical center of Guangdong Medical University Affiliated Second Hospital. The information we collected included all parts of the NoSAS score, the ESS, the STOP-Bang questionnaire and the Berlin questionnaire. Based on the severity of SDB determined by the apnea-hypopnea index (AHI), the patients were classified into four groups of primary snoring ( $<5$ events/h), mild SBD (AHI $\geq 5$ and $<15$ events/h), moderate SBD (AHI $\geq 15$ and $\leq 30$ events/h) and severe SBD ( $>30$ events/h). We calculated the sensitivity, specificity, positive predictive value, negative predictive value and area under the receiver operating characteristic curve of the five questionnaires to compare their relative efficacy for screening SDB.

Results: A total of 479 consecutive patients (374 males and 105 females) ranging in age from 18 to 80 years old (mean $\pm \mathrm{SD}, 48.9 \pm 14.4$ years old) were recruited into this study. When using the standard of $\mathrm{AHI} \geq 5$ for diagnosing SDB, the NoSAS score had the largest area under the curve (AUC) (AUC =0.734), and the Berlin questionnaire (AUC $=0.732$ ) came second. Both exhibited a better predictive value than the ESS score and the STOP-Bang questionnaire. Using NoSAS $\geq 8$ to predict AHI $\geq 5$ events/h, AHI $\geq 15$ events/h and $\mathrm{AHI}>30$ events/h, the sensitivity and specificity were 0.590 and $0.707,0.649$ and 0.626 , and 0.644 and 0.562 , respectively; for the STOP-Bang questionnaire, the values were 0.721 and $0.512,0.752$ and 0.440 , and 0.763 and 0.399, respectively; and for the Berlin questionnaire, the values were 0.721 and $0.512,0.752$ and 0.440 , and 0.763 and 0.399 , respectively.

Conclusions: The NoSAS score and the Berlin questionnaire both exhibited good predictive value for SDB patients. NoSAS is a more suitable questionnaire to use in clinic for the conveniences but the similar performance with another questionnaire. 
Keywords: NoSAS score; Epworth Sleepiness Scale (ESS); sleep-disordered breathing (SDB); STOP-Bang questionnaire; Berlin questionnaire

Submitted Dec 21, 2017. Accepted for publication Jun 25, 2018.

doi: $10.21037 /$ jtd.2018.07.46

View this article at: http://dx.doi.org/10.21037/jtd.2018.07.46

\section{Introduction}

Sleep-disordered breathing (SDB) is a systemic disease with many causes. SDB is characterized by repeated disruptions of breathing during sleep, which can cause intermittent hypoxia, hypercapnia, and sleep fragmentation and thus has many potential consequences, including cardiopulmonary and cerebrovascular diseases and metabolic effects (1). It has been suggested that $2 \%$ of women and $4 \%$ of adult men have SDB (2), but in recent years, the prevalence rates of overweight and obesity experienced epidemic trajectories in the United States (3-5), which is likely to have resulted in increased occurrence of obesity-related outcomes, including SDB. Some studies have found that the incidence of SDB is far greater than that. Peppard et al. found that approximately $34 \%$ of men and $17 \%$ of women are, to different extents, affected by SDB (6). Currently, polysomnography (PSG) is the gold standard for diagnosing SDB, but it is limited in wide application, especially in primary hospitals with limited economic conditions, because it is timeconsuming, complex, expensive and requires professional sleep monitoring sites and professional and technical personnel. Therefore, it is necessary to apply a simple and effective screening tool for patients at high risk for SDB. Currently, the scales used for screening SDB are the Berlin questionnaire (7), the STOP-Bang questionnaire (8), and the Epworth Sleepiness Scale (9), which can subjectively evaluate the severity of daytime sleepiness in SDB patients (10). The NoSAS score is a new screening tool first used by Marti-Soler et al. in a study to screen for SDB in a cohort study of 2,121 subjects based on the Swiss population. The tool was proven effective by a cohort study in Brazil (EPISONO) that included 1,042 subjects. The two cohorts of this study proved that the NoSAS score can be used as a simple and effective screening tool for screening patients suspected of sleep apnea (11), but the research was based on a population-based sample without relevant clinical research. Prof. Guichard et al. (12), Tan et al. (13) and Qing et al. (14) found that NoSAS has good application value both in the research based on the population-based sample and hospital-based sample. The NoSAS score, which ranges from 0 to 17 , includes 5 questions, allocating 4 points for having a neck circumference of more than $40 \mathrm{~cm}, 3$ points for having a body mass index (BMI) of $25 \mathrm{~kg} / \mathrm{m}^{2}$ to less than $30 \mathrm{~kg} / \mathrm{m}^{2}$ or 5 points for having a body mass index of $30 \mathrm{~kg} / \mathrm{m}^{2}$ or more, 2 points for snoring, 4 points for being older than 55 years of age, and 2 points for being male. The NoSAS score identifies individuals at risk of clinically significant SDB using a threshold of 8 points. The ESS score, which includes 8 questions, asks respondents to rate their sleepiness in eight daily situations from 0 to 3 , resulting in a total score of 0 (no daytime sleepiness) to 24 (the most excessive daytime sleepiness), with a threshold of 9 or more for daytime sleepiness. The STOP-Bang questionnaire, based on the STOP questionnaire, has four additional indexes called "Bang": B (BMI $\left.>35 \mathrm{~kg} / \mathrm{m}^{2}\right)$, A (>50 years old), N (neck circumference $>40 \mathrm{~cm}), \mathrm{G}$ (male). It adds 1 point when the answer is "yes" and 0 points when "no". The STOP-Bang questionnaire indicates that the patients are at high risk for obstructive sleep apnea (OSA) when the total points are greater than 3. The Berlin questionnaire has been widely used as an SDB screening tool worldwide, and it includes 11 questions on three topics: (I) the severity of snoring; (II) daytime sleepiness; and (III) high blood pressure or obesity. The assessment of each topic is negative or positive. If the assessment of 2 or more topics is positive, then the patient is considered to have a high risk for SDB (highrisk group). Our study retrospectively analyzed the existing data of patients suspected of SDB in the Second Affiliated Hospital of Guangdong Medical University, comparing the effectiveness of the NoSAS score, the ESS, the STOP-Bang questionnaire, and the Berlin questionnaire in screening for SDB to evaluate the application value of NoSAS scores in patients with SDB.

\section{Methods}

This study was a retrospective analysis of patients who had 
already had a PSG examination between October 2012 and October 2017 at the Second Hospital Sleep Medicine Center Affiliated with the Guangdong Medical University. The inclusion criteria were age greater than 18 years old, independent behavioral and cognitive abilities, the ability to answer the questionnaires completely and complete anthropometric data. Exclusion criteria included a history of brain tumors or epilepsy, benzodiazepine use, and treatment for SDB.

In our study, we collected basic data retrospectively from existing data, including age, sex, occupation, education, and measurement indicators such as height, weight, neck circumference, and waist circumference from all the patients with a diagnosis of sleep apnea. Data recorded during the patients' sleep included snoring and apnea and their severity and duration.

\section{PSG monitoring}

We monitored the patients with Alice 5 PSG (Philips) for at least $7 \mathrm{~h}$. Alcohol, coffee, sedatives and hypnotics were prohibited on the day of the test. The monitoring indicators included peripheral blood oxygen saturation, electrooculogram, electro-encephalogram, electromyography, electrocardiogram, snoring, nasal airflow, chest breathing, and body position. Data were analyzed according to the 2012 American Academy of Sleep Medicine (AASM) (15) guidelines for the scoring of sleep and associated events by sleep professionals and technical personnel to evaluate sleep stages and sleep-related breathing events and finally corrected by the same doctor. Referring to the American College of Physicians (ACP) guidelines for the diagnosis and treatment of obstructive sleep apnea syndrome (16), the patients were defined as having SDB when the apneahypopnea index (AHI) was not fewer than 5 events/h. The patients were classified into four groups based on the AHI: the pure snoring group $(\mathrm{AHI}<5$ events/h), the mild SDB group (AHI $\geq 5$ and $<15$ events/h), the moderate SDB group (AHI $\geq 15$ and $\leq 30$ events/h), and the severe SDB group (AHI $>30$ events/h).

\section{Statistical analysis}

Statistical analyses were performed using the SPSS v16.0. The normally distributed data are presented as the mean $\pm \mathrm{SD}$, and mean values were compared by single factor variance analysis. The comparison between the two groups was analyzed by post hoc analysis. The count data are expressed as rates. The $\chi^{2}$ test was used to compare categorical variables. Tests were considered significant at $\mathrm{P}<0.05$. Receiver operating characteristic (ROC) curve was used to analyze the diagnostic performance of the four scales.

\section{Results}

A total of 479 consecutive patients $(374$ males and 105 females) ranging in age from 18 to 80 years old (mean \pm SD, $48.9 \pm 14.4$ years old; Table 1 ) were recruited into this study (Figure 1). The baseline characteristics of the 479 patients suspected of having SDB who were enrolled in our study, including 374 male cases (78.1\%), are presented in Table 1. The mean age of the subjects was $48.9 \pm 14.4$ years old, the mean BMI was $25.9 \pm 4.3 \mathrm{~kg} / \mathrm{m}^{2}$, and the mean neck and waistline circumferences were $36.9 \pm 4.1$ and $91.8 \pm 11.5 \mathrm{~cm}$, respectively. The mean ESS, NoSAS, Berlin, and STOP-Bang scores were 7.8 \pm 5.4 , $7.9 \pm 3.7,1.5 \pm 0.9$, and $3.2 \pm 1.3$ points, respectively. The prevalence rates of the pure snoring group, mild SDB group, moderate SDB group and severe SDB group were 164 cases (34.2\%), 113 cases (23.6\%), 84 cases (17.5\%), and 118 cases $(24.6 \%)$, respectively. There were no statistically significant differences in age $(\mathrm{P}=0.089)$ among the groups, whereas the differences among BMI, neck circumference, waist circumference, ESS score, NoSAS score, STOP-Bang score and Berlin score were statistically significant $(\mathrm{P}<0.001)$ among the groups. The differences in the NoSAS score between the pure snoring group and mild, moderate, and severe SDB groups exhibited statistical significance $(\mathrm{P}<0.05)$; the differences between the mild SDB and moderate or severe SDB groups were statistically significant, whereas the difference between the medium and heavy SDB groups exhibited no statistical significance.

The effectiveness of screening with each scale was determined. Using AHI cutoffs of 5, 10, 15, 20, 25 and 30 events/h to compare the area under the curve (AUC) among the NoSAS score, the ESS score, the STOP-Bang score, and the Berlin score, we found (Table 2) that the AUC for the NoSAS score was highest when using the AHI cutoffs of 5, 10, 15 and 25 events/h. Meanwhile, the AUC for the ESS score was the lowest. Additionally, the predictive value of the NoSAS score $(\mathrm{AUC}=0.734$ ) was better than that of the ESS score (AUC $=0.615$ ), the STOPBang score (AUC $=0.702$ ) or the Berlin score $(\mathrm{AUC}=0.732$, Figure 2) when using $\mathrm{AHI} \geq 5$ events/h.

Using the cutoff point of AHI $\geq 5$ events/h (Table 3), 
Table 1 Baseline characteristics of the study subjects

\begin{tabular}{|c|c|c|c|c|c|c|}
\hline Index & Total & $\mathrm{AHI}<5$ & $5 \leq \mathrm{AHI}<15$ & $15 \leq \mathrm{AHI} \leq 30$ & $\mathrm{AHI}>30$ & $\mathrm{P}$ \\
\hline Male, n (\%) & $374(78.1)$ & $112(30.0)$ & $88(23.5)^{\mathrm{a}}$ & $67(17.9)^{\mathrm{ab}}$ & $107(28.6)^{\mathrm{bc}}$ & $<0.001$ \\
\hline Age (years) & $48.9 \pm 14.4$ & $48.6 \pm 16.1$ & $50.2 \pm 12.4$ & $51.1 \pm 13.9$ & $46.4 \pm 13.7$ & 0.089 \\
\hline BMI (kg/m²) & $25.9 \pm 4.3$ & $24.0 \pm 4.0$ & $25.6 \pm 4.0^{\mathrm{a}}$ & $26.8 \pm 3.4^{\mathrm{a}}$ & $28.1 \pm 3.2^{\mathrm{ab}}$ & $<0.001$ \\
\hline Weight (kg) & $71.2 \pm 14.1$ & $61.6 \pm 13.1$ & $70.0 \pm 13.0^{a}$ & $74.0 \pm 11.8^{\mathrm{a}}$ & $79.7 \pm 12.9^{\mathrm{abc}}$ & $<0.001$ \\
\hline AHI (time/h) & $20.3 \pm 23.1$ & $1.8 \pm 1.5$ & $9.1 \pm 2.7^{\mathrm{a}}$ & $21.4 \pm 3.9^{\mathrm{ab}}$ & $55.9 \pm 16.7^{\mathrm{abc}}$ & $<0.001$ \\
\hline $\mathrm{SBP}(\mathrm{mmHg})$ & $128.5 \pm 16.3$ & $126.4 \pm 17.9$ & $126.7 \pm 16.0$ & $131.2 \pm 17.2$ & $131.0 \pm 13.0$ & 0.027 \\
\hline $\mathrm{DBP}(\mathrm{mmHg})$ & $76.4 \pm 12.1$ & $74.0 \pm 12.5$ & $74.5 \pm 11.3$ & $78.2 \pm 12.6$ & $80.1 \pm 11.0^{\mathrm{ab}}$ & $<0.001$ \\
\hline WC (cm) & $91.8 \pm 11.5$ & $85.9 \pm 10.6$ & $91.0 \pm 10.5^{a}$ & $95.8 \pm 9.9^{\mathrm{ab}}$ & $97.7 \pm 10.5^{\mathrm{ab}}$ & $<0.001$ \\
\hline ESS & $7.8 \pm 5.4$ & $6.4 \pm 5.0$ & $7.3 \pm 4.9$ & $7.5 \pm 5.1$ & $10.4 \pm 5.8^{\mathrm{abc}}$ & $<0.001$ \\
\hline NoSAS & $7.9 \pm 3.7$ & $5.9 \pm 3.4$ & $7.7 \pm 3.3^{\mathrm{a}}$ & $9.4 \pm 3.1^{\mathrm{ab}}$ & $9.9 \pm 3.6^{\mathrm{ab}}$ & $<0.001$ \\
\hline Berlin & $1.5 \pm 0.9$ & $0.9 \pm 0.8$ & $1.5 \pm 0.9^{\mathrm{a}}$ & $1.8 \pm 0.8^{\mathrm{ab}}$ & $2.0 \pm 0.8^{\mathrm{ab}}$ & $<0.001$ \\
\hline STOP-Bang & $3.2 \pm 1.3$ & $2.6 \pm 1.2$ & $3.2 \pm 1.2^{\mathrm{a}}$ & $3.8 \pm 1.4^{\mathrm{ab}}$ & $3.8 \pm 1.3^{\mathrm{ab}}$ & $<0.001$ \\
\hline $\mathrm{LNSpO}_{2}(\%)$ & $79.6 \pm 13.6$ & $88.6 \pm 6.7$ & $84.2 \pm 6.1^{\mathrm{a}}$ & $76.8 \pm 9.5^{\mathrm{ab}}$ & $85.0 \pm 15.6^{\mathrm{abc}}$ & $<0.001$ \\
\hline $\mathrm{MNSpO}_{2}(\%)$ & $94.4 \pm 3.5$ & $95.7 \pm 2.2$ & $95.3 \pm 1.9$ & $94.5 \pm 2.3^{\mathrm{a}}$ & $91.5 \pm 4.7^{\mathrm{abc}}$ & $<0.001$ \\
\hline
\end{tabular}

Data are presented as the mean \pm SD or number (percentage). ${ }^{a}$, compared with the group of $\mathrm{AHI}<5, \mathrm{P}<0.05 ;{ }^{\mathrm{b}}$, compared with the group of $5 \leq \mathrm{AHI}<15, \mathrm{P}<0.05$; $^{\circ}$, compared with the group of $15 \leq \mathrm{AHI} \leq 30, \mathrm{P}<0.05$. BMI, body mass index; AHI, apnea-hypopnea index; SBP, systolic pressure; DBP, diastolic blood pressure; NC, neck circumference; WC, waist circumference; $\mathrm{LNSpO}_{2}$, minimum nighttime oxygen saturation; $\mathrm{MNSpO}_{2}$, mean nighttime oxygen saturation.

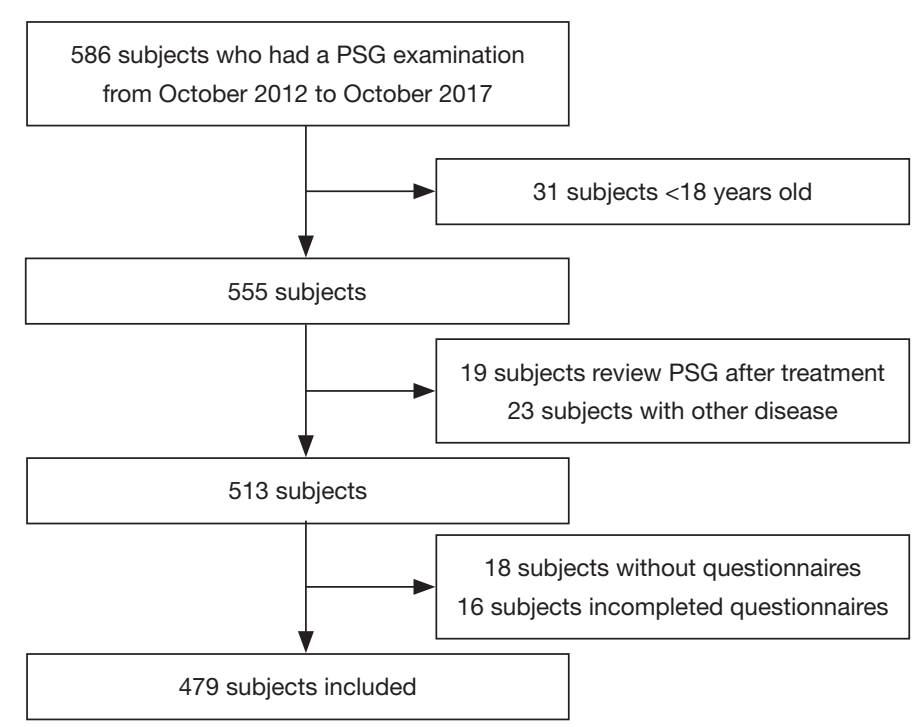

Figure 1 Flow diagram. PSG, polysomnography. 
Table 2 AUC (AHI $\geq 5,10,15,20,25,30$ events/h as the diagnostic criteria for SDB)

\begin{tabular}{lcccccc}
\hline Questionnaire & $\mathrm{AHI} \geq 5$ & $\mathrm{AHI} \geq 10$ & $\mathrm{AHI} \geq 15$ & $\mathrm{AHI} \geq 20$ & $\mathrm{AHI} \geq 25$ & $\mathrm{AHI}>30$ \\
\hline NoSAS & 0.734 & 0.733 & 0.731 & 0.707 & 0.706 & 0.688 \\
ESS & 0.615 & 0.614 & 0.623 & 0.640 & 0.662 & 0.670 \\
Berlin & 0.732 & 0.722 & 0.710 & 0.709 & 0.702 & 0.694 \\
STOP-Bang & 0.702 & 0.688 & 0.686 & 0.683 & 0.644 \\
\hline
\end{tabular}

Data are presented as values. AUC, area under the curve; AHI, apnea-hypopnea index; SDB, sleep-disordered breathing.

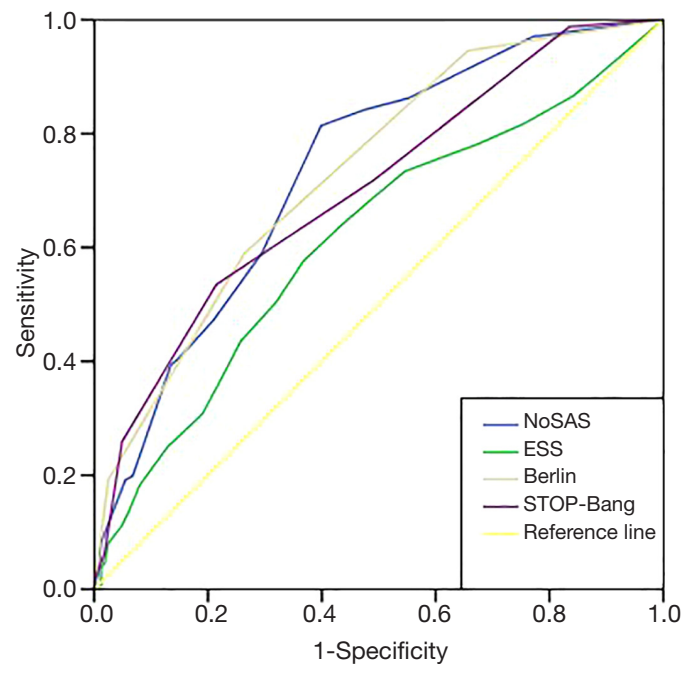

Figure 2 ROC curve (AHI $\geq 5$ events/h as the diagnostic criteria for SDB). ROC, receiver operating characteristic; AHI, apneahypopnea index; ESS, Epworth Sleepiness Scale.

the sensitivity of the STOP-Bang questionnaire in predicting SDB was the best (0.721), followed by the Berlin questionnaire and the NoSAS score (0.590). In addition, the specificity of the Berlin questionnaire was the highest (0.738), followed by the NoSAS score (0.707) and then the ESS. Using the cutoff points of AHI $\geq 15$ or 30 events/h (Tables 4,5), the results were similar.

\section{Discussion}

SDB can involve multiple systems to cause intermittent hypoxia, which harms the human body through oxidative stress and inflammatory reactions. Therefore, there is an urgent need to identify a more accurate, rapid way to screen patients for SDB. To our knowledge, there are many kinds of predictive scales to screen for SDB, including the Epworth scale, the Berlin questionnaire, the STOP-
Bang questionnaire, the ASA checklist and the sleep apnea questionnaire (17), but most of them are complex and not completely consistent with the actual situation in China, such as the question regarding falling asleep while waiting for the traffic light while driving in the Berlin questionnaire and the Epworth scale. Many Chinese patients cannot answer the questionnaire without driving experience, leading to the low feasibility of completion and poor accuracy in such scales. Abrishami et al. (18) showed that the STOP-Bang questionnaire not only has a high prediction efficiency but is also easy to use. However, its deficiencies include three subjective variables, including snoring, fatigue and observed apnea and $\mathrm{BMI}>35 \mathrm{~kg} / \mathrm{m}^{2}$. In this study, we found that the BMI of the severe SDB group $\left(28.1 \mathrm{~kg} / \mathrm{m}^{2}\right)$ was still lower than that of the STOP-Bang questionnaire and standard Berlin questionnaire, which has a cutoff value of $30 \mathrm{~kg} / \mathrm{m}^{2}$. $\mathrm{BMI}>30 \mathrm{~kg} / \mathrm{m}^{2}$ is an important scoring item of Berlin questionnaire, which means patient with $\mathrm{BMI}>30 \mathrm{~kg} / \mathrm{m}^{2}$ is more likely to be SDB. Compared with these scales, the thresholds for BMI in the NoSAS score were modified by adjusting the score for the different BMI values, with $25<\mathrm{BMI}<30 \mathrm{~kg} / \mathrm{m}^{2}$ assigned three points and $\geq 30 \mathrm{~kg} / \mathrm{m}^{2}$ assigned five points. Therefore, it is important to have appropriate scoring for BMI thresholds, which can influence the accuracy of the questionnaire. Additionally, there is only one subjective variable in the NoSAS score, snoring, which means that the NoSAS score has the minimum number of variables out of these five questionnaires, making it more convenient for clinicians to use.

The NoSAS score is a new screening tool that is a more credible scale developed by a large sample size cohort based on a community population in a foreign study by Marti-Soler et al. (11) and verified by a cohort study in a community in Brazil. In their study, using an AHI cutoff of $\geq 20$ events/h as the standard for diagnosing SDB, the authors found that the AUC of NoSAS was the largest and that the cutoff point of the highest predictive value was 8 . Comparing the capability of the NoSAS, Berlin, and STOP- 
Table $3 \mathrm{AHI} \geq 5$ events/h as the diagnostic criteria for SDB

\begin{tabular}{lcccc}
\hline Questionnaire & Sensitivity & Specificity & PPV & NPV \\
\hline NoSAS & $0.590(0.536-0.645)$ & $0.707(0.637-0.777)$ & $0.795(0.743-0.847)$ & $0.473(0.411-0.536)$ \\
ESS & $0.508(0.453-0.563)$ & $0.677(0.605-0.748)$ & $0.751(0.694-0.809)$ & $0.417(0.358-0.477)$ \\
Berlin & $0.590(0.536-0.645)$ & $0.738(0.670-0.805)$ & $0.812(0.761-0.862)$ & $0.484(0.422-0.546)$ \\
STOP-Bang & $0.721(0.671-0.770)$ & $0.512(0.436-0.589)$ & $0.739(0.690-0.788)$ & $0.488(0.414-0.563)$ \\
\hline
\end{tabular}

Data are presented as values (range). AHI, apnea-hypopnea index; SDB, sleep-disordered breathing; PPV, positive predictive value; NPV, negative predictive value.

Table $4 \mathrm{AHI} \geq 15$ events/h as the diagnostic criteria for SDB

\begin{tabular}{lcccc}
\hline Questionnaire & Sensitivity & Specificity & PPV & NPV \\
\hline NoSAS & $0.649(0.583-0.714)$ & $0.626(0.571-0.685)$ & $0.560(0.496-0.623)$ & $0.710(0.653-0.767)$ \\
ESS & $0.559(0.491-0.628)$ & $0.639(0.582-0.696)$ & $0.531(0.463-0.598)$ & $0.665(0.463-0.598)$ \\
Berlin & $0.644(0.578-0.710)$ & $0.643(0.586-0.699)$ & $0.568(0.504-0.632)$ & $0.712(0.656-0.768)$ \\
STOP-Bang & $0.752(0.693-0.812)$ & $0.440(0.382-0.499)$ & $0.493(0.439-0.551)$ & $0.709(0.641-0.777)$ \\
\hline
\end{tabular}

Data are presented as values (range). AHI, apnea-hypopnea index; SDB, sleep-disordered breathing; PPV, positive predictive value; NPV, negative predictive value.

Table $5 \mathrm{AHI}>30$ events/h as the diagnostic criteria for SDB

\begin{tabular}{lcccc}
\hline Questionnaire & Sensitivity & Specificity & PPV & NPV \\
\hline NoSAS & $0.644(0.558-0.730)$ & $0.562(0.511-0.614)$ & $0.325(0.265-0.385)$ & $0.829(0.781-0.876)$ \\
ESS & $0.634(0.545-0.723)$ & $0.613(0.563-0.663)$ & $0.333(0.270-0.397)$ & $0.846(0.802-0.889)$ \\
Berlin & $0.686(0.603-0.770)$ & $0.590(0.539-0.641)$ & $0.354(0.292-0.416)$ & $0.852(0.808-0.896)$ \\
STOP-Bang & $0.763(0.686-0.839)$ & $0.399(0.348-0.449)$ & $0.293(0.242-0.295)$ & $0.837(0.782-0.892)$
\end{tabular}

Data are presented as values (range). AHI, apnea-hypopnea index; SDB, sleep-disordered breathing; PPV, positive predictive value; NPV, negative predictive value.

Bang scores, the NoSAS score performed significantly better than the other scores, suggesting that the NoSAS questionnaire can be used as a simple and effective screening tool for patients with suspected SDB. It is important to screen for SDB in a community and to evaluate the severity of SDB. In an Asian cohort study produced by Tan et al. (13), the application efficiency of the NoSAS score was also high in screening the Asian population. There was no difference in the specificity among these three studies, but the sensitivity of the Tan et al.'s study was lower than those in the other two cohorts. Two studies $(19,20)$ previously reported that Chinese subjects have high rates of SDB despite relatively low BMIs. The authors suggested that the pathogenesis of SDB in the Chinese population may be related to some special factors, such as craniofacial restriction, neuromuscular control of the upper airway, or arousal threshold rather than obesity. SDB subjects who fail to be identified as high risk by the NoSAS score tend to have low BMIs and neck circumferences. As a result, some SDB subjects might not score 8 points on the NoSAS score if they have slim bodies. This may have an impact on our results. Therefore, using the cutoff point of $A H I \geq 5$ events/h, the sensitivity of the NoSAS score in predicting SDB was not the highest (0.590), followed by the STOP-Bang questionnaire (0.721), despite the fact that the NoSAS score has a high specificity (0.707).

We found that the morbidity of SDB in our 479 subjects was $65.8 \%$ (315 cases). The proportion of males (262/374, $70.1 \%$ ) in the SDB patients was significantly higher than that of women $(53 / 105,50.5 \%)$, which is consistent with the results of previous studies (21). 
The ideal screening scale should not only have a large AUC (22), but also should be highly sensitive to avoid false negative results and highly specific to avoid false positives. We used an AHI cutoff of $\geq 5$ events/h as the standard to diagnose SDB, making the AUC of the NoSAS score the largest (0.734), followed by the Berlin scale (0.732). All of the scales had AUC values above 0.7, which means they have moderate predictive values for SDB. However, the AUC of the ESS score and the STOP-Bang score were slightly worse (0.702 and 0.615 , respectively). This is the first time that using the ROC curve resulted in a larger AUC, which confirms that the NoSAS score had a good degree of differentiation for SDB patients. A previous study found that suspected SDB patients in the Caucasus region were ages 48 to 57 years old, and those in eastern Asian regions were 42 to 50 years old, suggesting that the age of SDB patients in eastern Asian regions is lower than that of SDB patients in the Caucasus region (23). SDB subjects who fail to be identified as low risk due to low BMI and neck circumference are identified by the NoSAS score. In our study, the age of the severe SDB group was lower than the threshold of the NoSAS score (55 years old). Compared with the Caucasian population, SDB patients in the domestic population have lower BMIs, a higher degree of severity, and earlier onset age.

This study had several advantages. Our study verified the application value of the NoSAS questionnaire in the clinical diagnosis of SDB patients. This study had some limitations. The patients in this study represented Chinese urban residents, and this study was a single-center retrospective study based on the Chinese population, which means that it was not representative of the general global population. Furthermore, the NoSAS score was gathered retrospectively from existing data, which may have an impact on the performance of the tool. Male patients made up a larger proportion of our study population, which may affect the results.

\section{Conclusions}

The NoSAS score and the Berlin questionnaire both exhibited good predictive value for SDB patients. Additionally, the NoSAS score is a simple, effective and practical SDB screening tool that can be widely used in screening in primary hospitals.

\section{Acknowledgements}

We thank Qizhong He and Tuxuan Liu (both the Second
Affiliated Hospital of Guangdong Medical University, Zhanjiang, China) for providing the questionnaire and the data.

Funding: This study was funded by the Natural Science Foundation of Guangdong Province (2014A030310219).

\section{Footnote}

Conflicts of Interest: The authors have no conflicts of interest to declare.

Ethical Statement: The study was approved by the Institutional Review Board (IRB number: 2018001) and written informed consent was obtained from all patients.

\section{References}

1. Zamarron C, García Paz V, Riveiro A, et al. Obstructive sleep apnea syndrome is a systemic disease. Current evidence. Eur J Intern Med 2008;19:390-8.

2. Coughlin SR, Mawdsley L, Mugarza JA, et al. Obstructive sleep apnoea is independently associated with an increased prevalence of metabolic syndrome. Eur Heart J 2004;25:735-41.

3. Flegal KM, Carroll MD, Ogden CL, et al. Prevalence and trends in obesity among US adults, 1999-2008. JAMA 2010;303:235-41.

4. Ogden CL, Carroll MD, Curtin LR, et al. Prevalence of overweight and obesity in the United States, 1999-2004. JAMA 2006;295:1549-55.

5. Hedley AA, Ogden CL, Johnson CL, et al. Prevalence of overweight and obesity among US children, adolescents, and adults, 1999-2002. JAMA 2004;291:2847-50.

6. Peppard PE, Young T, Barnet JH, et al, Increased prevalence of sleep-disordered breathing in adults. Am J Epidemiol 2013;177:1006-14.

7. Pataka A, Daskalopoulou E, Kalamaras G, et al. Evaluation of five different questionnaires for assessing sleep apnea syndrome in a sleep clinic. Sleep Med 2014;15:776-81.

8. Chung F, Yegneswaran B, Liao P, et al. STOP questionnaire: a tool to screen patients for obstructive sleep apnea. Anesthesiology 2008;108:812-21.

9. Johns MW. A new method for measuring daytime sleepiness: the Epworth Sleepiness Scale. Sleep 1991;14:540-5.

10. Tachibana N, Taniguchi M. why do we continue to use Epworth sleepiness scale? Sleep Med 2007;8:541-2.

11. Marti-Soler H, Hirotsu C, Marques-Vidal P, et al. The 
NoSAS score for screening of sleep-disordered breathing: a derivation and validation study. Lancet Respir Med 2016;4:742-8.

12. Guichard K, Marti-Soler H, Micoulaud-Franchi JA, et al. The NoSAS score: A new and simple screening tool for obstructive sleep apnea syndrome in depressive disorder. J Affect Disord 2018;227:136-40.

13. Tan A, Hong Y, Tan LW, et al. Validation of NoSAS score for screening of sleep-disordered breathing in a multiethnic Asian population. Sleep Breath 2017;21:1033-8.

14. Qing SM, Chen RK, Liu H, et al. Comparison of the NoSAS score with four different questionnaires as screening tools for obstructive sleep apnea-hypopnea syndrome. Zhonghua Jie He He Hu Xi Za Zhi 2018;41:213-9.

15. Berry RB, Budhiraja R, Gottlieb DJ, et al. Rules for scoring respiratory events in sleep: update of the 2007 AASM Manual for the Scoring of Sleep and Associated Events. Deliberations of the Sleep Apnea Definitions Task Force of the American Academy of Sleep Medicine. J Clin Sleep Med 2012;8:597-619.

16. Qaseem A, Holty JE, Owens DK, et al. Management of obstructive sleep apnea in adults: A clinical practice guideline from the American College of Physicians. Ann Intern Med 2013;159:471-83.

Cite this article as: Peng M, Chen R, Cheng J, Li J, Liu W, Hong C. Application value of the NoSAS score for screening sleep-disordered breathing. J Thorac Dis 2018;10(8):47744781. doi: 10.21037/jtd.2018.07.46
17. Ramachandran SK, Josephs LA. A meta-analysis of clinical screening tests for obstructive sleep apnea. Anesthesiology 2009;110:928-39.

18. Abrishami A, Khajehdehi A, Chung F. A systematic review of screening questionnaires for obstructive sleep apnea. Can J Anaesth 2010;57:423-38.

19. Lee RW, Vasudavan S, Hui DS, et al. Differences in craniofacial structures and obesity in Caucasian and Chinese patients with obstructive sleep apnea. Sleep 2010;33:1075-80.

20. Li KK, Kushida C, Powell NB, et al. Obstructive sleep apnea syndrome: a comparison between far-east Asian and white men. Laryngoscope 2000;110:1689-93.

21. Ip MS, Lam B, Tang LC, et al. A community study of sleep-disordered breathing in middle-aged Chinese women in Hong Kong: prevalence and gender differences. Chest 2004;125:127-34.

22. Silva GE, Vana KD, Goodwin JL, et al. Identification of patients with sleep disordered breathing: comparing the four-variable screening tool, STOP, STOP-Bang, and Epworth Sleepiness Scales. J Clin Sleep Med 2011;7:467-72.

23. Li Z, Du L, Li Y, et al. Characterization of primary symptoms leading to Chinese patients presenting at hospital with suspected obstructive sleep apnea. J Thorac Dis 2014;6:444-51. 